\title{
Magnetic detection under high pressures using designed silicon vacancy centers in silicon carbide
}

\section{Jun-Feng Wang}

University of Science and Technology of China https://orcid.org/0000-0002-5720-056X

Lin Liu

Institute of Solid State Physics, HFIPS, Chinese Academy of Sciences

Xiao-Di Liu

Institute of Solid State Physics

Qiang Li

University of Science and Technology of China

Jin-Ming Cui

University of Science and Technology of China

Di-Fan Zhou

Shanghai University

Ji-Yang Zhou

University of Science and Technology of China

Yu Wei

University of Science and Technology of China

\section{Hai-An Xu}

Institute of Solid State Physics, HFIPS, Chinese Academy of Sciences

\section{Wan Xu}

Institute of Solid State Physics, HFIPS, Chinese Academy of Sciences,

\section{Wu-Xi Lin}

University of Science and Technology of China

Jin-Wei Yan

Institute of Solid State Physics, HFIPS, Chinese Academy of Sciences

\section{Zhen-Xuan He}

University of Science and Technology of China

\section{Zheng-Hao Liu}

University of Science and Technology of China https://orcid.org/0000-0001-8067-1477

\section{Zhi-He Hao}

University of Science and Technology of China

\section{Hai-Ou Li}

University of Science and Technology of China

Wen Liu

University of Science and Technology of China

Jin-Shi Xu

University of Science and Technology of China https://orcid.org/0000-0003-0528-1000

\section{Eugene Gregoryanz}

School of Physics and Centre for Science at Extreme Conditions https://orcid.org/0000-0001-9080-8756

Chuan-Feng Li ( $\nabla$ cfli@ustc.edu.cn )

University of Science and Technology of China https://orcid.org/0000-0001-6815-8929

\section{Guangcan Guo}

University of Science and Technology of China 
Letter

Keywords:

Posted Date: February 21st, 2022

DOI: https://doi.org/10.21203/rs.3.rs-1308876/v1

License: (c) (i) This work is licensed under a Creative Commons Attribution 4.0 International License. Read Full License 


\section{Abstract}

Pressure is a significant parameter to investigate the properties of matter. The phenomenon of pressure-induced magnetic phase transition has attracted great interest due to its ability to detect superconducting behaviour at high pressures in diamond anvil cell. However, detection of the local sample magnetic properties is a great challenge due to the small sample chamber volume. Recently, in situ pressure-induced phase transition has been detected using optically detected magnetic resonance (ODMR) method of the nitrogen vacancies (NV) centers in diamond. However, the NV centers with four orientation axes and temperature-dependent zero-field-splitting present some difficulty to interpret the observed ODMR spectra. Here, we investigate and characterize the optical and spin properties of the implanted silicon vacancy defects in $4 \mathrm{H}-\mathrm{SiC}$, which is single-axis and temperature-independent zero-field-splitting. Using this technique, we observe the magnetic phase transition of a magnetic $\mathrm{Nd}_{2} \mathrm{Fe}_{14} \mathrm{~B}$ sample at about $7 \mathrm{GPa}$. Finally, the critical temperaturepressure phase diagram of the superconductor $\mathrm{YBa}_{2} \mathrm{Cu}_{3} \mathrm{O}_{6.66}$ is mapped and refined. These experiments pave the way for the silicon vacancy-based quantum sensor being used in situ magnetic detection at high pressures.

\section{Introduction}

Pressure can alter electronic, magnetic and lattice properties of matter, which is vital both in fundamental and applied sciences ${ }^{1-4}$. The high pressure techniques can be applied in many fields including physics, material science, geophysics and chemistry ${ }^{1}$. Plethora of exciting and important phenomenona were observed under pressure ${ }^{1-4}$. Particularly, pressure-induced high critical temperature $\left(T_{c}\right)$ superconductivity has drawn much attention in recently ${ }^{1-4}$. For example, lanthanum hydride has been demonstrated to be a superconductor with a critical temperature of around $250 \mathrm{~K}$ at pressures of about $170 \mathrm{GPa}^{4}$. One of the great challenges of superconducting studies at high pressure is the measurement of magnetic properties and their evolution. However, conventional methods such as using superconducting quantum interference devices (SQUID) or the AC susceptibility cannot directly detect weak magnetic signals at microscale inside the diamond anvil cell (DAC) sample chamber ${ }^{5-11}$. Therefore, it is urgent to explore new methods for the magnetic detection.

Recently, the high sensitivity and high resolution of the in situ magnetic detections in the DAC chamber was achieved by utilising the nitrogen-vacancy (NV) centres ${ }^{7-9}$. Diamond NV centers are versatile solid-state spin quantum sensors, which have been used to detect a wide variety of physical parameters such as magnetic field, electric field, temperature, strain, spins, and so on ${ }^{12}$. The zero-fieldsplitting (ZFS) parameter D of the NV centre ground spin state was shown to linearly increased as the pressure with a slope of 14.6 $\mathrm{MHz} / \mathrm{GPa}$ up to $60 \mathrm{GPa}^{6}$. On this basis, in situ magnetic detection method based on NV centers through the optically detected magnetic resonance (ODMR) technologies had been developed at high-pressure recently ${ }^{7-10}$. Micron-sized diamonds with ensemble NV centers have been placed inside the DAC chamber to measure $\mathrm{T}_{\mathrm{c}}$-pressure phase diagram of a superconductor ${ }^{7}$ and detect the pressureinduced magnetic phase transition of a magnet ${ }^{10}$. On the other hand, shallow implanted NV centers in the surface of the DAC culet are also used to probe the magnetization of a Fe particle and the Meissner effect of a superconductor, and construct the full stress-tensor on the culet surface ${ }^{8,9}$. Besides the ODMR methods, the noise spectroscopy method has been used to probe the phase transition of a gadolinium ${ }^{9}$.

However, NV centers have four different orientation axes in the diamond crystal, and the corresponding ODMR signals have eight resonate peaks, making it difficult to analyse the ODMR spectrum of the specific axis under a magnetic field ${ }^{7-10}$. It will be especially intractable for using diamond particles in the DAC chamber due to the unknown and different orientations of the NV centers ${ }^{7,10}$. Additionly, the ZFS parameter D decreases with the temperature from around $50 \mathrm{~K}$ to $600 \mathrm{~K}^{13,14}$, meanwhile, it also increases with the pressure at the same time, the convolution in P-T space present some some difficulty to interpret the observed spectra. Defects in silicon carbide $(\mathrm{SiC})$ is another promising candidate to measure magnetic properties under extreme conditions. We then use a similar solid quantum sensor based on silicon vacancy $\left(\mathrm{V}_{\mathrm{Si}}\right)$ defects in $4 \mathrm{H}-\mathrm{SiC}$ to realize the in situ magnetic measurement under high pressure.

$\mathrm{SiC}$ is a widely used semiconductor due to its unique properties, such as mature inch-scale growth and micro-nano fabrication ${ }^{15-17}$. In recent years, several spin qubits and bright single-photon-emitters in SiC attracted great attentions in the quantum community ${ }^{15-26}$. Particularly, the $\mathrm{V}_{\mathrm{Si}}(\mathrm{V} 2)$ defect has been extensively used in spin-photon interface ${ }^{18}$, quantum photonics ${ }^{21}$, quantum information processing ${ }^{17}$, quantum sensing such as magnetic field ${ }^{27}$ and temperature ${ }^{28,29}$ due to its outstanding properties. its spin state is $S=3 / 2$ spin quartet and the ground state ZFS parameter $\mathrm{D}$ is $70 \mathrm{MHz}{ }^{17,18}$. In contrast to NV centers in diamond, it only has one axis (along the 
c-axis of the $4 \mathrm{H}-\mathrm{SiC})$, and the corresponding ODMR spectrum only has two resonate peaks under an external magnetic field, which is convenient to readout the resonate frequencies and enhance the scalability in SiC devices ${ }^{17,29}$. Moreover, the ZFS parameter $D$ is also almost temperature independent from $20 \mathrm{~K}$ to $500 \mathrm{~K}$, which is beneficial to temperature-pressure research ${ }^{28,29}$. Besides, comparison with the traditional high-pressure magnetometry techniques, the $\mathrm{V}_{\mathrm{Si}}$ defects detected spatial resolution is only about a few microns.

Here, we investigate and characterize the optical and spin properties of the implanted silicon vacancy defects in $4 \mathrm{H}-\mathrm{SiC}$, which is singleaxis and with temperature-independent zero-field-splitting. The experimental results show that the PL spectrum has a blue shift and the ZFS parameter $\mathrm{D}$ increases with pressure at the rate $0.31 \mathrm{MHz} / \mathrm{GPa}$ under the pressure. Based on this, we have probed the pressureinduced magnetic phase transition of a $\mathrm{Nd}_{2} \mathrm{Fe}_{14} \mathrm{~B}$ magnet at about $7 \mathrm{GPa}$ at room temperature. Finally, Meissner effect of the superconductor $\mathrm{YBa}_{2} \mathrm{Cu}_{3} \mathrm{O}_{6.66}$ at different pressures is observed and we map and refine the $\mathrm{T}_{\mathrm{c}}-\mathrm{P}$ phase diagram. These experiments demonstrate the feasibility of using $\mathrm{V}_{\mathrm{Si}}$ defects in $\mathrm{SiC}$ as novel quantum sensors and open up the applications to study superconducting phenomena under extreme conditions

\section{Results}

Two high-quality single crystal $4 \mathrm{H}-\mathrm{SiC}$ cubes are used to fabricate $\mathrm{SiC}$ anvil cells using the standard design with $200 \mu \mathrm{m}$ diameter culets. As shown in Fig. 1a, high-density shallow $\mathrm{V}_{\mathrm{Si}}$ defects in a $100 \mathrm{~nm}$ depth layer are used for magnetic detection ${ }^{30}$ (see details in Methods). A non-magnetic rhenium gasket is used to confine the sample between the two anvils ${ }^{8}$. The $\mathrm{NaCl}$ is applied as the pressure transmitting medium in all the experiments. The in situ pressure is monitored by measuring the PL spectrum of the ruby (about $10 \mu \mathrm{m})$ in the pressure chamber. A $10 \mu \mathrm{m}$ platinum wire is placed across the culet surface, which is used to transmit microwave to control $\mathrm{V}_{\mathrm{Si}}$ defects spin states.

The energy levels of $\mathrm{V}_{\mathrm{Si}}$ defects at high pressures are shown in Fig. 1b. The $720 \mathrm{~nm}$ laser pumps the electron from the ground state to the phonon sideband, and the ZPL at ambient pressure is $916 \mathrm{~nm}$. Both the ZPL and the ground spin state ZFS parameter D change under high pressure. We first investigate the optical properties of the $\mathrm{V}_{\mathrm{Si}}$ defects at high pressures. The details of experimental setup can be found in Methods. The room temperature PL spectra of $V_{S i}$ defects and the corresponding ruby PL spectra at three different pressures are presented in Fig. 1c. The PL spectra of the ruby are used to monitor the local pressure. While, the PL spectra of $\mathrm{V}_{\mathrm{Si}}$ defects shift towards a shorter wavelength with the pressure, which is similar to that of NV center in diamond ${ }^{6}$. We then study mean counts of the $V_{S i}$ defects as a function of pressure. As shown in Fig. 1d, the counts increase as the pressure increases from the ambient pressure to around $8 \mathrm{GPa}$, which is due to the higher detect efficiency at the shorter wavelength of the single-photon counting module. Then the counts decrease as the pressure increase to around $25 \mathrm{GPa}$ (see Supplementary Note 1 for more details). The decrease of the photon counts may due to the lattice distortion of the $4 \mathrm{H}-\mathrm{SiC}$ caused by compression.

The characterization of $\mathrm{V}_{\mathrm{Si}}$ defect spin properties at high pressures is the foundation for high-pressure magnetic field sensing. Three respectively ODMR spectra at zero external magnetic field are presented in Fig. 2a. The resonant frequency shifts to higher frequencies as the pressure increases, which is similar to the ODMR signal of NV centers in diamond ${ }^{6-11,31,32}$. The reason might due to the local structural distortions and the decreasing distance between $\mathrm{V}_{\mathrm{Si}}$ spins in the macroscopic compression at the corresponding SiC lattices ${ }^{6,31,32}$. As shown in Fig. 2b, the mean ZFS parameter $D$ increases linearly with the pressure with the coefficient of $0.31 \pm 0.01$ $\mathrm{MHz} / \mathrm{GPa}$, which is much less than the $14.6 \mathrm{MHz} / \mathrm{GPa}$ of the NV centers in diamond $\mathrm{d}^{6,10,11}$. The smaller slope is beneficial for directly observing the shift of the ODMR signal with a large pressure range. The reason for the small slope might be due to the degeneracy of half-integer $V_{S i}$ defects $(S=3 / 2)$, which makes it rather insensitive to fluctuations in strain ${ }^{33}$. Through the coherent control of $V_{S i}$ defects, the dynamic magnetic properties of the magnetic materials without a direct magnetic signal could be detected ${ }^{9,10}$. Figure $2 \mathrm{c}$ shows the measurement of the Rabi oscillation at ambient pressure using a standard pulse sequence ${ }^{29}$. Inferred from the fitting, the Rabi frequency is about $9 \mathrm{MHz}$. Then the coherence time under high pressures are studied. Figures $2 \mathrm{~d}$ and $2 \mathrm{e}$ present the spin echo measurement of $\mathrm{V}_{\mathrm{Si}}$ defects at

Fig. 2 The spin properties of $\mathbf{V}_{\mathrm{si}}$ defects at high pressures. $\mathrm{a}$, The ODMR spectra at three different pressures with zero external magnetic field. The solid lines represent Lorentzian fittings. b. The mean ZFS parameter D linearly increases with the pressure up to 27 GPa. Error bars represent the standard deviations of the measured results. c, Rabi oscillation at 31 Gauss at ambient pressure. The red line is fitted using an exponential decaying sine function. $\mathbf{d}$ and $\mathbf{e}$, The spin echo results at ambient pressure and $15.1 \mathrm{GPa}$, respectively. 
Red lines represent the exponentially decay fittings to the data. $\mathbf{f}$, The coherence time $\mathrm{T}_{2}$ as a function of the pressure. Error bars are the data fitting standard deviations.

ambient pressure and $15.1 \mathrm{GPa}$, respectively. The corresponding coherence time $\mathrm{T}_{2}$ are $7.8 \pm 0.9 \mu$ s and $7.8 \pm 0.8 \mu$ s, respectively. Both the values are consistent with previous results ${ }^{29}$. The coherence time $\mathrm{T}_{2}$ as a function of pressure up to $25 \mathrm{GPa}$ are summarized in Fig. $2 \mathrm{f}$. The coherence time remains invariable up to $25 \mathrm{GPa}$, which is similar to that of $\mathrm{NV}$ centers in diamond ${ }^{10}$. The robustness $\mathrm{V}_{\mathrm{Si}}$ defects coherence time under pressure helps detect phase transitions of magnetic samples at high pressures without an obvious static magnetic signals ${ }^{9,10}$.

Pressure-induced magnetic phase transitions of a common magnet $\mathrm{Nd}_{2} \mathrm{Fe}_{14} \mathrm{~B}$ sample at room temperature aredetectedhere using the ODMR methods of the $\mathrm{V}_{\mathrm{Si}}$ defects. A small piece of $\mathrm{Nd}_{2} \mathrm{Fe}_{14} \mathrm{~B}$ sample is placed on the surface of the culets. The PL image of the implanted shallow $\mathrm{V}_{\mathrm{Si}}$ defects and $\mathrm{Nd}_{2} \mathrm{Fe}_{14} \mathrm{~B}$ sample on the culet surface are presented in Fig.3a. In order to efficiently detect the magnetic field of the sample, a location close to the sample (black dashed line region) is chosen as the detect position, which is denoted as a black cross. As a comparison, a remote location is the reference position (denoted as a red cross). In the experiment, we apply a c-axis (perpendicular to the culet) magnetic field $B_{c}$ with the strength of 198 Gauss. Three schematics of local magnetic field vectors at the detected position under different pressures are shown in Fig. $3 \mathrm{~b}$. The $\mathrm{B}_{\mathrm{C}}, \mathrm{B}_{\mathrm{NdFeB}}$ and $\mathrm{B}_{\text {tot }}$ represent the $\mathrm{c}$-axis external magnetic field, magnetic field of the $\mathrm{Nd}_{2} \mathrm{Fe}_{14} \mathrm{~B}$ sample and total magnetic field on the $\mathrm{V}_{\mathrm{Si}}$ defects, respectively. The strength of $\mathrm{B}_{\mathrm{NdFeB}}$ decreases with pressure in the compression processes and it recovers in the decompression processes. It leads to the corresponding changes of the ODMR spectra signal as the pressure. We calculate the magnetic field of the $\mathrm{Nd}_{2} \mathrm{Fe}_{14} \mathrm{~B}$ sample by the $\left|\mathrm{B}_{\text {tot }} \mathrm{B}_{\mathrm{C}}\right| \mathrm{B}$. Both of the $B_{\text {tot }}$ and $B_{c}$ can be deduced from the measured ODMR spectra at each pressure. The representative several ODMR signals at the detected positions during the compression process are presented in Fig.3c. The ODMR signals at the reference position reflecting the strength of external magnetic field are also measured at each pressure. One of representative ones at the pressure 0.3 GPa is shown in Fig.3c. The ODMR resonant frequencies at the detected position do not change up to $5.1 \mathrm{GPa}$, and then they increase quickly up to 6.7 GPa. Finally, they again slightly change as the pressure further increases. The summary of the magnetic field of the sample measured by $\mathrm{V}_{\mathrm{Si}}$ defects during the compression (blue squares) and decompression (red dots) processes are shown in Fig.4d, respectively. In the compression process, the sample magnetic field decreases slowly as the pressure increases to about 6 GPa, then it has a sharp reduction in the range of about 6-8 GPa. Finally, it slightly decreases as the pressure increases to around $9.5 \mathrm{GPa}$. This phenomenon demonstrates that the $\mathrm{Nd}_{2} \mathrm{Fe}_{14} \mathrm{~B}$ sample changes from ferromagnetic phase to paramagnetic phase at around $7 \mathrm{GPa}$, which is consistent with previous results ${ }^{10}$. Furthermore, we also measure the sample magnetic field during the decompression process, which is almost the same as that in the compression process. It is due to the robust magnetic property of the used $\mathrm{Nd}_{2} \mathrm{Fe}_{14} \mathrm{~B}$ sample. $\mathrm{The}$ magnetic field-pressure data demonstrate the pressure-induced ferromagnetic-paramagnetic phase transitions of the $\mathrm{Nd}_{2} \mathrm{Fe}_{14} \mathrm{~B}$ sample in the range of 6 to $10 \mathrm{GPa}$, which is also consistent with previous results ${ }^{10,34}$.

Figure 3 The detection of pressure-induced magnetic phase transition of a $\mathrm{Nd}_{2} \mathrm{Fe}_{14} \mathrm{~B}$ magnet using the shallow $\mathrm{V}$ si defects. a, The PL image of the $\mathrm{V}_{\mathrm{Si}}$ defects and $\mathrm{Nd}_{2} \mathrm{Fe}_{14} \mathrm{~B}$ sample in the culet surface. The black and red crosses are the detected and reference positions, respectively. The black dashed line region indicates the $\mathrm{Nd}_{2} \mathrm{Fe}_{14} \mathrm{~B}$ sample. The scale bar is $20 \mu \mathrm{m}$. b, Three typically local magnetic fields vectors during the pressure-induced magnetic phase transition in the compression and decompression processes. The external magnetic field $\mathrm{B}_{\mathrm{c}}$ is along the $\mathrm{c}$-axis of the $4 \mathrm{H}$-SiC. The magnetic field of the $\mathrm{Nd}_{2} \mathrm{Fe}_{14} \mathrm{~B}$ sample and the total magnetic field are labelled as $B_{N d F e B}$ and $B_{\text {tot }}$, respectively. $c$, The shallow $V_{S i}$ defects ODMR spectra in the detected and reference positions in the culet surface during the compression process. $d$, The inferred magnetic fields of the $\mathrm{Nd}_{2} \mathrm{Fe}_{14} \mathrm{~B}$ sample measured by $\mathrm{V}_{\mathrm{Si}}$ defects during the compression (blue squares) and decompression (red circles) processes, respectively.

In the past decades, extreme conditions were applied to synthesize and study the novel superconducting materials ${ }^{1-4,7-9}$. It has been used to provide new insights to the mechanism of superconducting and explore higher $\mathrm{T}_{\mathrm{C}}$ superconductors ${ }^{1-4,7-9}$. As a proof-ofprincipleexperiment, we detect the superconducting phase transition of the well-known superconductor $\mathrm{YBa}_{2} \mathrm{Cu}_{3} \mathrm{O}_{\mathrm{x}}{ }^{35-38}$ using the implanted $\mathrm{V}_{\mathrm{Si}}$ defects at different pressures and low temperature. $\mathrm{YBa}_{2} \mathrm{Cu}_{3} \mathrm{O}_{\mathrm{x}}$ is a type II high- $\mathrm{T}_{\mathrm{C}}$ (liquid nitrogen temperature range) superconductor, with different concentration of oxygen $\mathrm{x}$. The $\mathrm{YBa}_{2} \mathrm{Cu}_{3} \mathrm{O}_{6.6}$ are used in the experiment due to its high $\mathrm{T}_{\mathrm{C}}$ and dramatically $\mathrm{T}_{c}$-pressure curve ${ }^{35}$. The $\mathrm{YBa}_{2} \mathrm{Cu}_{3} \mathrm{O}_{6.6}$ sample is made by conventional heat treatment methods (see Supplementary Note 2 for more details). In the experiment, we put a tiny flake of $\mathrm{YBa}_{2} \mathrm{Cu}_{3} \mathrm{O}_{6.6}$ on the surface of the culet. Then the SiC-based pressure 
chamber is set in the cryostat ${ }^{31}$. The confocal scanning image of $\mathrm{V}_{\mathrm{Si}}$ defects and $\mathrm{YBa}_{2} \mathrm{Cu}_{3} \mathrm{O}_{6.66}$ sample on the culet is delineated in Fig.4a. We detected the Meissner effect of the sample (black dashed line region) through the ODMR spectra at the detected position (black cross) under the pressure of $9.0 \mathrm{GPa}^{6-8}$. In order to measure superconductor magnetic moment, we first cool the superconductor below its $\mathrm{T}_{\mathrm{c}}$ in zero magnetic field, then a small c-axis magnetic field (7.7 Gauss) is applied to generate a Zeeman splitting of the $\mathrm{V}_{\mathrm{Si}}$ defects $^{37-39}$. The ODMR measurements are performed as the temperature increases. The ODMR spectra with respect to temperatures are shown in Fig.4b, which have different splittings at different temperatures. This phenomenon indicates the diamagnetism associated with superconductivity Meissner effect. The summary of the ODMR splitting as a function of temperature are presented in Fig.4c. The splitting almost does not change as the temperature increases from $82.4 \mathrm{~K}$ to $94.7 \mathrm{~K}$, but then it has a step-like jump at around $95 \mathrm{~K}$. Then the splitting remains nearly invariable as the temperature further increases to $108 \mathrm{~K}$. The red line represents the fitting of the data using a sigmoid function: $S(T)=a+b /\left(1+\exp \left[-\left(T-T_{c}\right) / \delta T_{c}\right)\right.$ ), where $a, b, \delta T_{c}$ are fitting parameters and $T_{c}$ is the critical temperature ${ }^{37,38}$. The fitted critical temperature $T_{c}$ at $9 \mathrm{GPa}$ is $95.3 \pm 0.2 \mathrm{~K}$, which is consistent with the previous results ${ }^{35}$. The small change of the ODMR splitting is consistent with the result measured by implanted NV centers in diamond, which may due to the large distance between the quantum sensors and the samples ${ }^{8}$.

Figure 4 The measurement of temperature-pressure phase diagram of the superconductor $\mathrm{YBa}_{2} \mathrm{Cu}_{3} \mathrm{O}_{6.66}$ using implanted $\mathrm{V}_{\mathrm{Si}}$ defects. a, The confocal scanning image of $\mathrm{YBa}_{2} \mathrm{Cu}_{3} \mathrm{O}_{6.66}$ sample and $\mathrm{V}_{\mathrm{Si}}$ defects in the culet surface. The black dashed line region marks the investigated $\mathrm{YBa}_{2} \mathrm{Cu}_{3} \mathrm{O}_{6.66}$ sample and the black cross marks the corresponding detected position. The size scale bar is $20 \mu \mathrm{m}$. $\mathbf{b}$, The ODMR spectra with the superconductivity diamagnetism in the detected position at different temperatures at $9.0 \mathrm{GPa}$. The dashed lines are guides to an eye only. c, The inferred ODMR splitting during the sample superconducting phase transition at $9.0 \mathrm{GPa}$. The red line is the fitting of the data. The superconducting transition temperature $T_{C}$ is deduced to be $95.2 \pm 0.2 \mathrm{~K}$. $d$, The inferred ODMR splittings as a function of the temperature under different pressures. The labeled coefficients are the ODMR splitting magnification times in order to normalization the data. $\mathbf{e}, \mathrm{The} \mathrm{YBa}_{2} \mathrm{Cu}_{3} \mathrm{O}_{6.66} \mathrm{~T}_{\mathrm{c}}$-pressure phase diagram. The $\mathrm{T}_{\mathrm{C}}$ under ambient pressure (blue dot) is measured through a magnetic property measurement system (see Supplementary Note 2 for more details). The $T_{c}$ under pressures (red dots) are inferred from the ODMR splittings. The shadow area represents the superconducting state and the transparent area is the normal state for the $\mathrm{YBa}_{2} \mathrm{Cu}_{3} \mathrm{O}_{6.66}$. The error bars are obtained from the fitting standard deviations are smaller than the symbol sizes.

We further investigate the critical temperatures at different pressures. Figure $4 \mathrm{~d}$ shows the measured ODMR splitting as a function of the temperature at different pressures, from which we can infer the pressure-dependent $T_{c}$. The critical temperature $T_{c}$ increases as the pressure increases. As presented in Fig. $4 \mathrm{e}$, the $\mathrm{YBa}_{2} \mathrm{Cu}_{3} \mathrm{O}_{6.66} \mathrm{~T}_{c}$-pressure phase diagram is mapped by the ODMR method. $\mathrm{T}_{c}$ increases quickly as the pressure increases from ambient pressure to about $12.3 \mathrm{GPa}$, then it increases slowly up to around $17.1 \mathrm{GPa}$, which are consistent with previous results ${ }^{35}$. The pressure dependence of $\mathrm{T}_{\mathrm{C}}$ is due to that high pressure leads to the change of charge carrier concentration in the $\mathrm{CuO}_{2}$ planes within the unit cell ${ }^{36}$. The shadow and transparent areas represent the superconducting state and normal state for the $\mathrm{YBa}_{2} \mathrm{Cu}_{3} \mathrm{O}_{6.66}$, respectively, which is in agreement with the previous results ${ }^{35}$. Furthermore, the $\mathrm{V}_{\mathrm{Si}}$ defects-based ODMR methods can be used to study the $T_{c}$-pressure phase diagram of $\mathrm{YBa}_{2} \mathrm{Cu}_{3} \mathrm{O}_{\mathrm{x}}$ with different concentration of oxygen $\mathrm{x}$ and its intrinsic properties, including the lower critical field and London penetration depth under high pressure $\mathrm{e}^{38,39}$.

\section{Discussion}

In conclusion, we realize in situ magnetic detection of the magnetic materials using implanted $\mathrm{V}_{\mathrm{Si}}$ defects ensemble in SiC-based anvil cells under high pressures. Through studying the optical and spin properties of the implanted $V_{S i}$ defects, the experiments show that the $\mathrm{PL}$ spectrum has a blue shift and the mean counts decrease under high pressure. At the same time, the ZFS parameter $D$ increases as the pressure with a small coefficient of $0.31 \mathrm{MHz} / \mathrm{GPa}$, which is much less than that of the NV centers in diamond. Moreover, the spin coherence time keeps invariable as the pressure which is vital to probe phase transitions of samples at high pressure without a direct magnetic signal. Besides, the ODMR contrast can further increase several times using optimized anneal parameters, which can improve the ODMR signal detecting effciency ${ }^{40}$. Based on these, the pressure-induced magnetic phase transitions of magnet $\mathrm{Nd}_{2} \mathrm{Fe}_{14} \mathrm{~B} \mathrm{sample}$ are detected in the range of 6-10 GPa using the ODMR methods at room temperature. Finally, we map and refine the superconductor $\mathrm{YBa}_{2} \mathrm{Cu}_{3} \mathrm{O}_{6.66} \mathrm{~T}_{\mathrm{c}}$-pressure phase diagram by the ODMR technology at low temperatures.

It can further realize the directly magnetic field image of the sample using a CMOS camera detector ${ }^{8}$. The $4 \mathrm{H}-\mathrm{SiC}$ micrometer particle with $\mathrm{V}_{\mathrm{Si}}$ defects ${ }^{41}$ and other types of spin qubit including divacancy ${ }^{15,16,42}$, NV center ${ }^{24-26}$ and even transition metal ions ${ }^{43}$ in $4 \mathrm{H}, 6 \mathrm{H}$ 
and $3 \mathrm{C}$ polytypes of $\mathrm{SiC}$ may also be applied to local magnetic detection at high pressure. Some types of novel spin readout technologies such as photocurrent detected magnetic resonance ${ }^{44,45}$ and anti-Stokes excited ODMR technology ${ }^{29}$ can also be used for $\mathrm{V}_{\mathrm{Si}}$ defects-based magnetic sensing under high pressure. The experiments form a framework for using SiC $\mathrm{V}_{\mathrm{Si}}$ defects in the local in situ magnetic detection under high pressure.

\section{Methods}

Silicon vacancy generation. In the experiments, two high-quality single crystal $4 \mathrm{H}-\mathrm{SiC}$ cubes are used to fabricate $\mathrm{SiC}$ anvil cells using the standard design. The culet diameter is $200 \mu \mathrm{m}$ and the crystalline orientation with the 1000 (c-axis). $20 \mathrm{keV}$ helium ions ( $\mathrm{He}^{+}$) with a dose of $1 \times 10^{13} / \mathrm{cm}^{2}$ are perpendicularly implanted to the culets to generate high-density shallow $\mathrm{V}_{\mathrm{Si}}$ defects and the corresponding depth is around $100 \mathrm{~nm}$ through the stopping and range of ions in matter (SRIM) simulation ${ }^{30}$. We then anneal the SiC anvil cells at $500{ }^{\circ} \mathrm{C}$ for 2 hours to further increase the $V_{S i}$ defects density by around 3 times ${ }^{30}$.

Experimental set-up. The measurement setup is a homebuilt confocal scan microscope equipped with a microwave system ${ }^{29.30}$. Two lasers with the wavelengths of $532 \mathrm{~nm}$ and $720 \mathrm{~nm}$ are used to excite the ruby and $V_{\mathrm{Si}}$ defects, respectively. Two $650 \mathrm{~nm}$ and $850 \mathrm{~nm}$ long-pass filters are used for the collection of ruby and $\mathrm{V}_{\mathrm{Si}}$ defects fluorescence, respectively. We adopt a long-working distance (20 $\mathrm{mm}$ ) infrared objective (0.4 N.A., Mitutoyo) to excite the samples and collect the fluorescence. A single-photon counting module (SPCM$A Q R H-14)$ is applied to the fluorescence of $V_{S i}$ defects to determine the average photon counts. A liquid nitrogen temperature range optical cryostat (Oxford Instruments) combined with a confocal system is applied in the low-temperature experiments ${ }^{46}$. The standard

lock-in technology is used to detect the ODMR and the coherence control signals using a photoreceiver (Femto, OE-200-Si) ${ }^{15,29}$. A c-axis (perpendicular to the culet surface) magnetic field $B_{c}$ is added to adjust the spin state energy levels.

\section{Declarations}

\section{Acknowledgements}

We thank Gang-Qin Liu, En-Ke Liu in Institute of Physics, Chinese Academy of Sciences for their helpful discussion. This work was supported by the National Key Research and Development Program of China (Grant No. 2016YFA0302700 and 2017YFA0304100), the National Natural Science Foundation of China (Grants No. U19A2075, 11975221, 11874361, 51672279, 51727806, 11774354, 61905233, 61725504, 11804330, 11821404 and 11774335), Science Challenge Project (No. TZ2016001), CAS Innovation Grant (No. CXJJ-19-B08), and the CASHIPS Director's Fund (Grant No. YZJJ201705). Anhui Initiative in Quantum Information Technologies (AHY060300 and AHY020100), the Fundamental Research Funds for the Central Universities (Grant No. WK2030380017). X. D. Liu are grateful for the support from Youth Innovation Promotion Association of CAS (No. 2021446), J. F. Wang also acknowledges financial support from the Science Specialty Program of Sichuan University (Grand No. 2020SCUNL210). This work was partially carried out at the USTC center for Micro and Nanoscale Research and Fabrication.

\section{Author contributions}

J.-F.W., X.-D. L. and J.-S.X. designed the experiments. J.-F.W. and L.L. built the experiment set-up and performed the measurements with the help of X.-D. L., Q.L., J.-Y.Z., J.-M.C., H.-A. X., W. X., J.-W. Y., W.-X.L., Z.-X.H., Z.-H.L., Z.-H.H., and H.-O.L.. L. L., J.-F.W., and X.-D.L. prepared the samples in the SiC-based high pressure chamber. D.-F.Z. prepared the YBCuO sample. Y.W. and W.L. preformed the implantation of the $\mathrm{V}_{\mathrm{Si}}$ defects. J.-F.W., J.-S.X., L.L. and X.-D.L. performed the data analysis with contributions from all co-authors. J.F.W., J.-S.X., X.-D.L. and E.G. wrote the manuscript with contributions from all co-authors. J.-S.X., X.-D.L., E.G., C.-F.L. and G.-C.G. supervised the project. All authors contributed to the discussion of the results.

\section{References}

1. Mao, H. K. et al. Solids, liquids, and gases under high pressure. Rev. Mod. Phys. 90, 015007 (2018).

2. Flores-Livas, J. A., et al. A perspective on conventional high-temperature superconductors at high pressure: Methods and materials. Phys. Rep., 856, 1-78 (2020). 
3. Drozdov, A. P. et al. Conventional superconductivity at 203 kelvin at high pressures in the sulfur hydride system, Nature, 525, 73-76 (2015).

4. Drozdov, A. P. et al. Superconductivity at $250 \mathrm{~K}$ in lanthanum hydride under high pressures. Nature 569, $528-531$ (2019).

5. Jayaraman, A. Diamond anvil cell and high-pressure physical investigations. Rev. Mod. Phys. 55, 65 (1983).

6. Doherty, M. W. et al. Electronic properties and metrology applications of the diamond NV'center under pressure. Phys. Rev. Lett. 112, 047601 (2014).

7. Yip, K. Y. et al. Measuring magnetic field texture in correlated electron systems under extreme conditions, Science 366,1355 (2019).

8. Lesik, M. et al. Magnetic measurements on micrometer-sized samples under high pressure using designed NV centers. Science 366, 1359 (2019).

9. Hsieh, S. et al. Imaging stress and magnetism at high pressures using a nanoscale quantum sensor. Science 366, 1349 (2019).

10. Shang, Y. X. et al. Magnetic Sensing inside a Diamond Anvil Cell via Nitrogen-Vacancy Center Spins. Chin. Phys. Lett. 36, 086201 (2019).

11. Ho, K. O. et al. Probing local pressure environment in anvil cells with nitrogen-vacancy (N-V) centers in diamond, Phys. Rev. Appl. 13, 024041 (2020).

12. Schirhagl, R. et al. Nitrogen-vacancy centers in diamond: nanoscale sensors for physics and biology. Annu. Rev. Phys. Chem. 65 , 83-105 (2014).

13. Chen, X. D. et al. Temperature dependent energy level shifts of nitrogen-vacancy centers in diamond. Appl. Phys. Lett. $\mathbf{9 9 ,} 161903$ (2011).

14. Toyli, D. et al. Measurement and control of single nitrogen-vacancy center spins above 600 K. Phys. Rev. X 2, 031001 (2012).

15. Koehl, W. F. et al. Room temperature coherent control of defect spin qubits in silicon carbide. Nature 479, 84-87 (2011).

16. Christle, D. J. et al. Isolated electron spins in silicon carbide with millisecond coherence times. Nat. Mater. 14, 160-163 (2015).

17. Widmann, M. et al. Coherent control of single spins in silicon carbide at room temperature. Nat. Mater. 14, 164-168 (2015).

18. Nagy, R. et al. High-fidelity spin and optical control of single silicon-vacancy centres in silicon carbide. Nat. Commun. 10, 1954 (2019)

19. Wang, J. F. et al. Bright room temperature single photon source at telecom range in cubic silicon carbide. Nat. Commun. 9, 4106 (2018).

20. Lohrmann, A., Johnson, B. C., McCallum, J. C. \& Castelletto, S. A review on single photon sources in silicon carbide. Rep. Prog. Phys. $80,034502(2017)$.

21. Lukin, D. M. et al. 4H-silicon-carbide-on-insulator for integrated quantum and nonlinear photonics. Nat. Photonics $14,330-334$ (2020).

24. Zargaleh, S. A. et al. Nitrogen vacancy center in cubic silicon carbide: a promising qubit in the $1.5 \mu \mathrm{m}$ spectral range for photonic quantum networks. Phys. Rev. B 98, 165203 (2018).

25. Wang, J. F. et al. Coherent control of nitrogen-vacancy center spins in silicon carbide at room temperature. Phys. Rev. Lett. 124, 223601 (2020).

26. $\mathrm{Mu}$, Z. et al. Coherent manipulation with resonant excitation and single emitter creation of nitrogen vacancy centers in $4 \mathrm{H}$ silicon carbide. Nano Lett. 20, 6142-6147 (2020). 
27. Simin, D. et al. All-optical dc nanotesla magnetometry using silicon vacancy fine structure in isotopically purified silicon carbide. Phys. Rev. X 6, 031014 (2016).

28. Anisimov, A. N. et al. Optical thermometry based on level anticrossing in silicon carbide. Sci. Rep. 6, 33301 (2016).

29. Wang, J. F. Robust coherent control of solid-state spin qubits using anti-Stokes excitation. Nat. Commun. 12, 3223 (2021).

30. Wang, J. F. et al. On-demand generation of single silicon vacancy defects in silicon carbide. ACS Photon. 6, 1736-1743 (2019).

31. Steele, L. G. et al. Optically detected magnetic resonance of nitrogen vacancies in a diamond anvil cell using designer diamond anvils. Appl. Phys. Lett. 111, 221903 (2017).

32. Ivády, V. et al. Pressure and temperature dependence of the zero-field splitting in the ground state of NV centers in diamond: A firstprinciples study. Phys. Rev. B 90, 235205 (2014).

33. Nagy, R. et al. Quantum properties of dichroic silicon vacancies in silicon carbide. Phys. Rev. Appl. 9, 034022 (2018).

34. Kamarád, J., Arnold, Z. and Schneider, J. Effect of pressure on the curie and spin reorientation temperatures of polycrystalline Nd2Fe14B compound. J. Magn. Magn. Mater. 67, 29 (1987).

35. Sadewasser, S., Schilling, J. S., Paulikas, A. P. and Veal B. W. Pressure dependence of Tc to $17 \mathrm{GPa}$ with and without relaxation effects in superconducting $\mathrm{YBa}_{2} \mathrm{Cu}_{3} \mathrm{O}_{\mathrm{x}}$. Phys. Rev. B. 61, 741 (2000).

36. Chen, X. J., Lin, H. Q. and Gong. C. D. Pressure Dependence of Tc in Y-Ba-Cu-O Superconductors. Phys. Rev. Lett. 85,2180 (2000).

37. Waxman, A. et al. Diamond magnetometry of superconducting thin films. Phys. Rev. B. 89, 054509 (2014).

38. Joshi, K.R. et al. Measuring the lower critical field of superconductors using nitrogen-vacancy centers in diamond optical magnetometry. Phys. Rev. Appl. 11, 014035 (2019).

39. Nusran, N. M. et al. Spatially-resolved study of the Meissner effect in superconductors using NV-centers-in-diamond optical magnetometry. New J. Phys. 20, 043010 (2018).

40. Abraham, J. B. S. et al. Nanotesla magnetometry with the silicon vacancy in silicon carbide. Phys. Rev. Applied 15, 064022 (2021).

41. Castelletto, S. et al. Fluorescent color centers in laser ablated 4H-SiC nanoparticles. Opt. Lett. 42, 1297 (2017).

42. Falk, A. L. et al. Polytype control of spin qubits in silicon carbide. Nat. Commun. 4, 1819 (2013).

43. Wolfowicz, G. et al. Vanadium spin qubits as telecom quantum emitters in silicon carbide. Sci. Adv. 6, eaaz1192 (2020).

44. Niethammer, M. et al. Coherent electrical readout of defect spins in silicon carbide by photo-ionization at ambient conditions. Nat. Commun. 10, 5569 (2019).

45. Ho, K. O. et al. Recent developments of quantum sensing under pressurized environment using the nitrogen vacancy (NV) center in diamond. J. Appl. Phys. 129, 241101 (2021).

46. Liu, X. D. et al. Counterintuitive effects of isotopic doping on the phase diagram of H2-HD-D2 molecular alloy. Proceedings of the National Academy of Sciences, 117, 8736-8742 (2020).

\section{Figures}


a

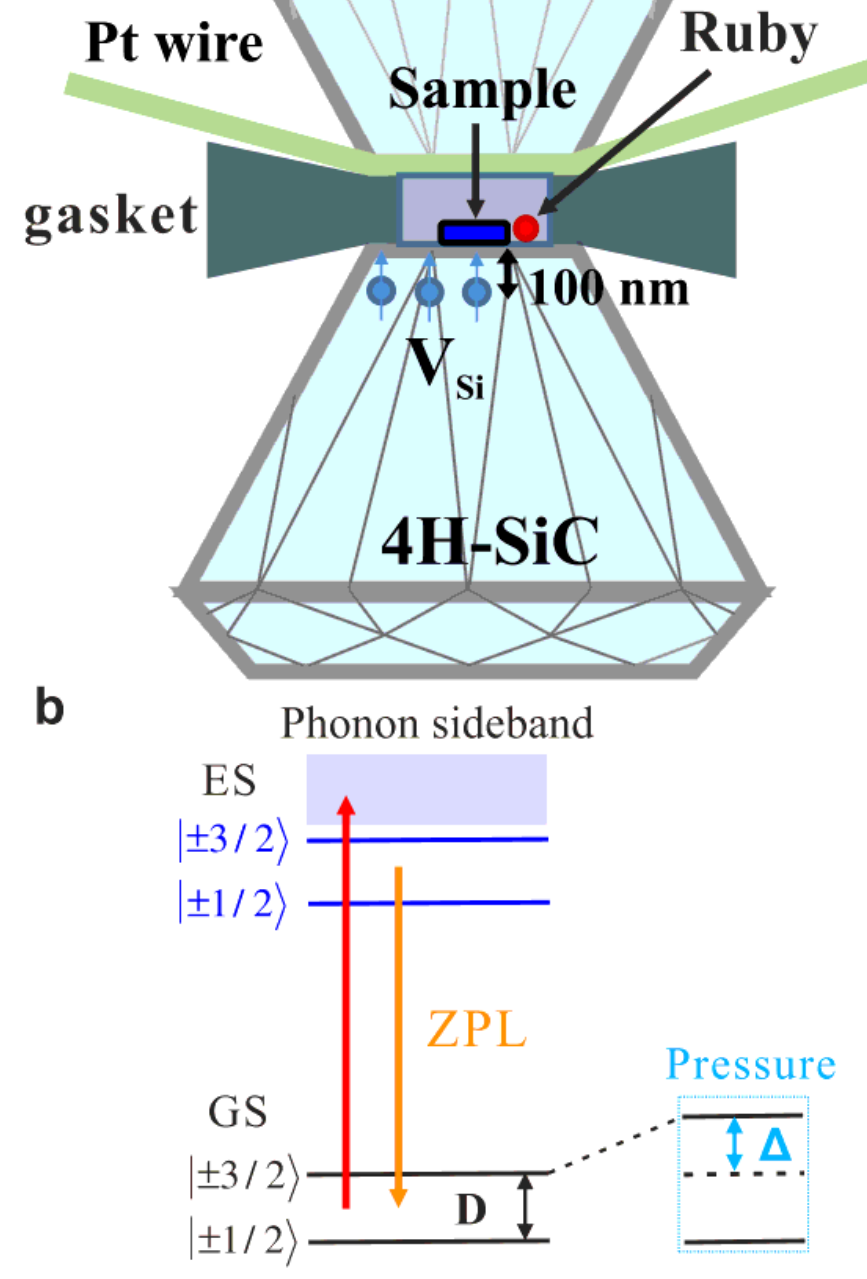

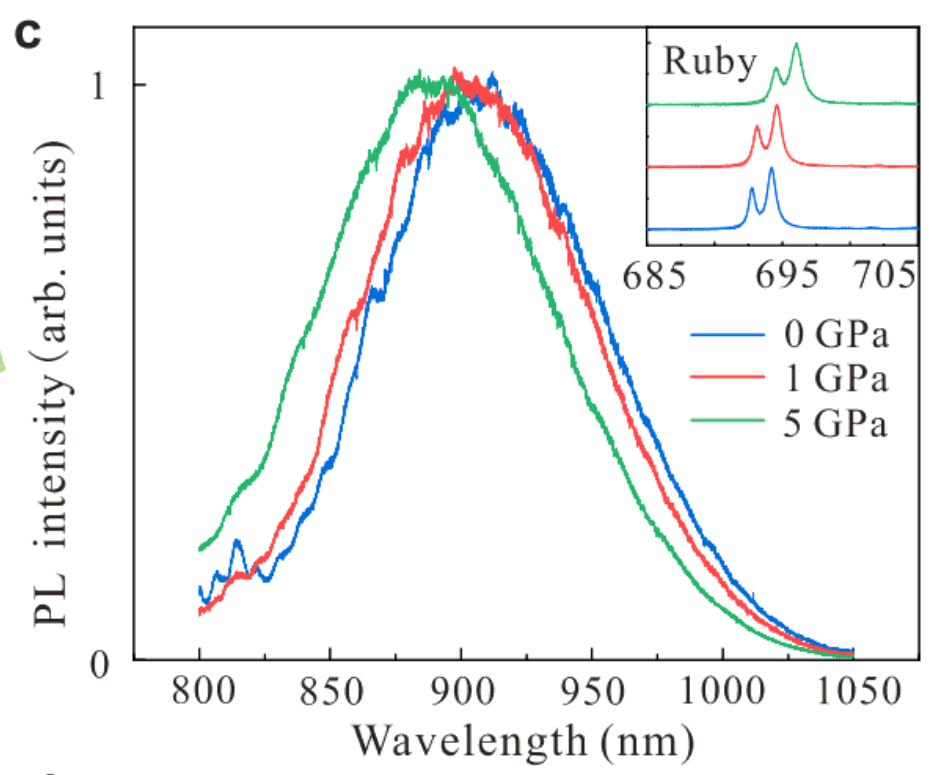

d

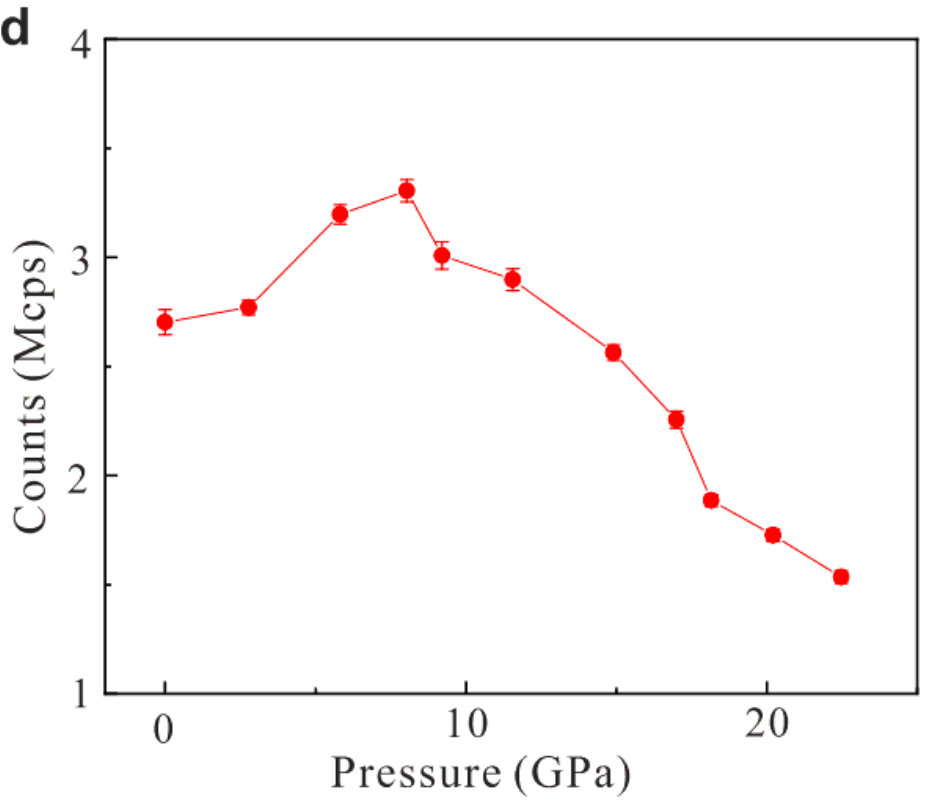

\section{Figure 1}

The SiC anvil cell and $\mathrm{V}_{\mathrm{Si}}$ defects optical properties with pressure. a, The schematic of SiC anvil cell. The samples are placed on the surface of the culet, and the local shallow $(100 \mathrm{~nm}) \mathrm{V}_{\mathrm{Si}}$ defects are used for the in situ magnetic detection. A $10 \mu \mathrm{m}$ ruby is put close to the samples to measure the pressure. $b$, Energy levels of $V_{S i}$ defects at high pressure. The red line indicates the $720 \mathrm{~nm}$ excitation laser. The pressure changes the ZPL emission and shifts the ground state ZFS parameter $D$ with $\Delta$. c, Room temperature PL spectra of the $V_{S i}$ defects at three representative pressures. The inset is the corresponding PL spectra of the ruby. $d$, The mean counts of $V_{S i}$ defects with $10 \mathrm{~mW}$ laser excitation as a function of the pressure. Error bars are due to the standard deviations of counts in an $8 \times 8 \mu \mathrm{m}^{2}$ area. 

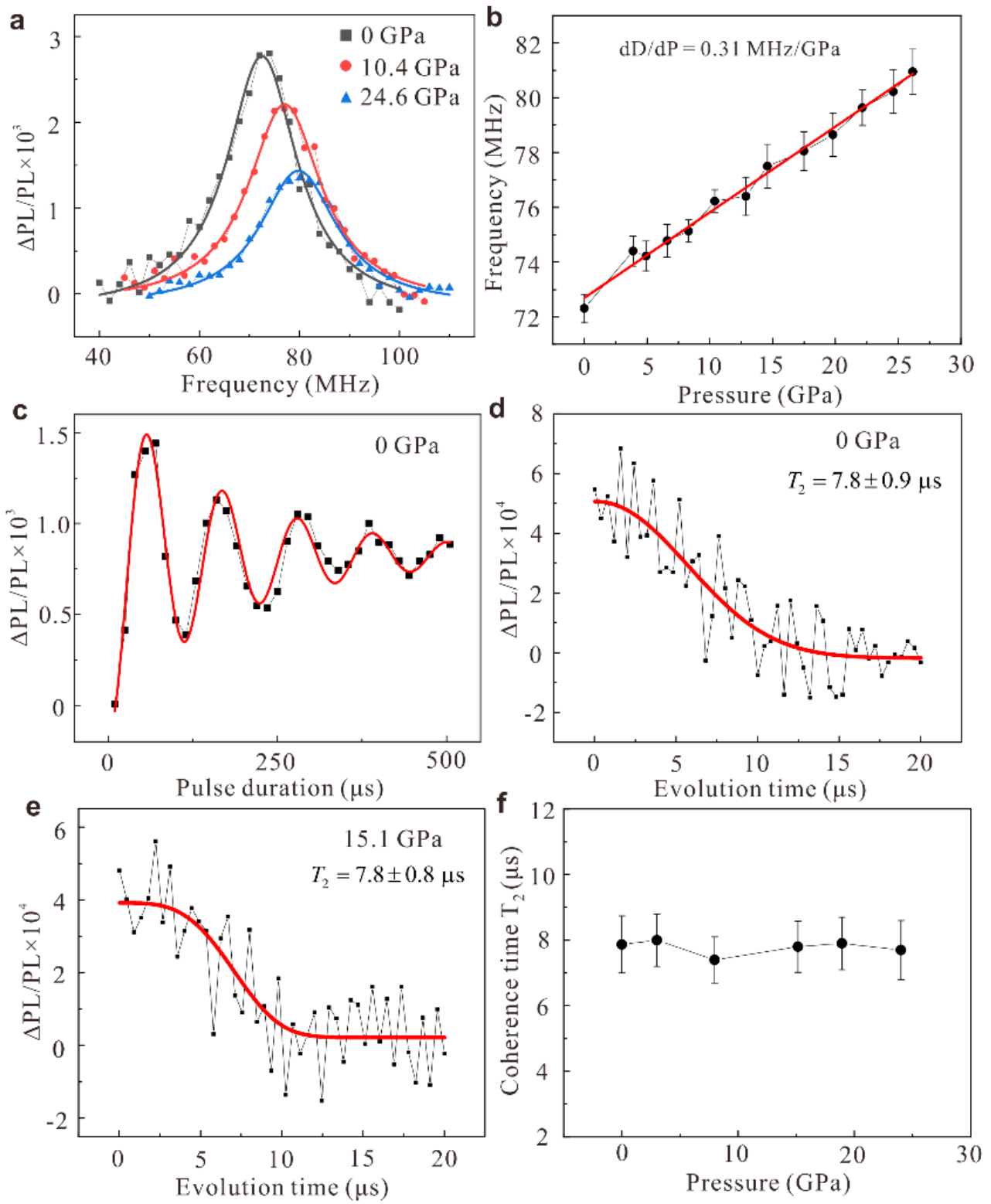

\section{Figure 2}

The spin properties of $\mathrm{V}_{\mathrm{Si}}$ defects at high pressures. $\mathrm{a}$, The ODMR spectra at three different pressures with zero external magnetic field. The solid lines represent Lorentzian fittings. b, The mean ZFS parameter D linearly increases with the pressure up to $27 \mathrm{GPa}$. Error bars represent the standard deviations of the measured results. c, Rabi oscillation at 31 Gauss at ambient pressure. The red line is fitted using an exponential decaying sine function. $\mathbf{d}$ and $\mathbf{e}$, The spin echo results at ambient pressure and $15.1 \mathrm{GPa}$, respectively. Red lines represent the exponentially decay fittings to the data. $\mathbf{f}$, The coherence time $\mathrm{T}_{2}$ as a function of the pressure. Error bars are the data fitting standard deviations. 

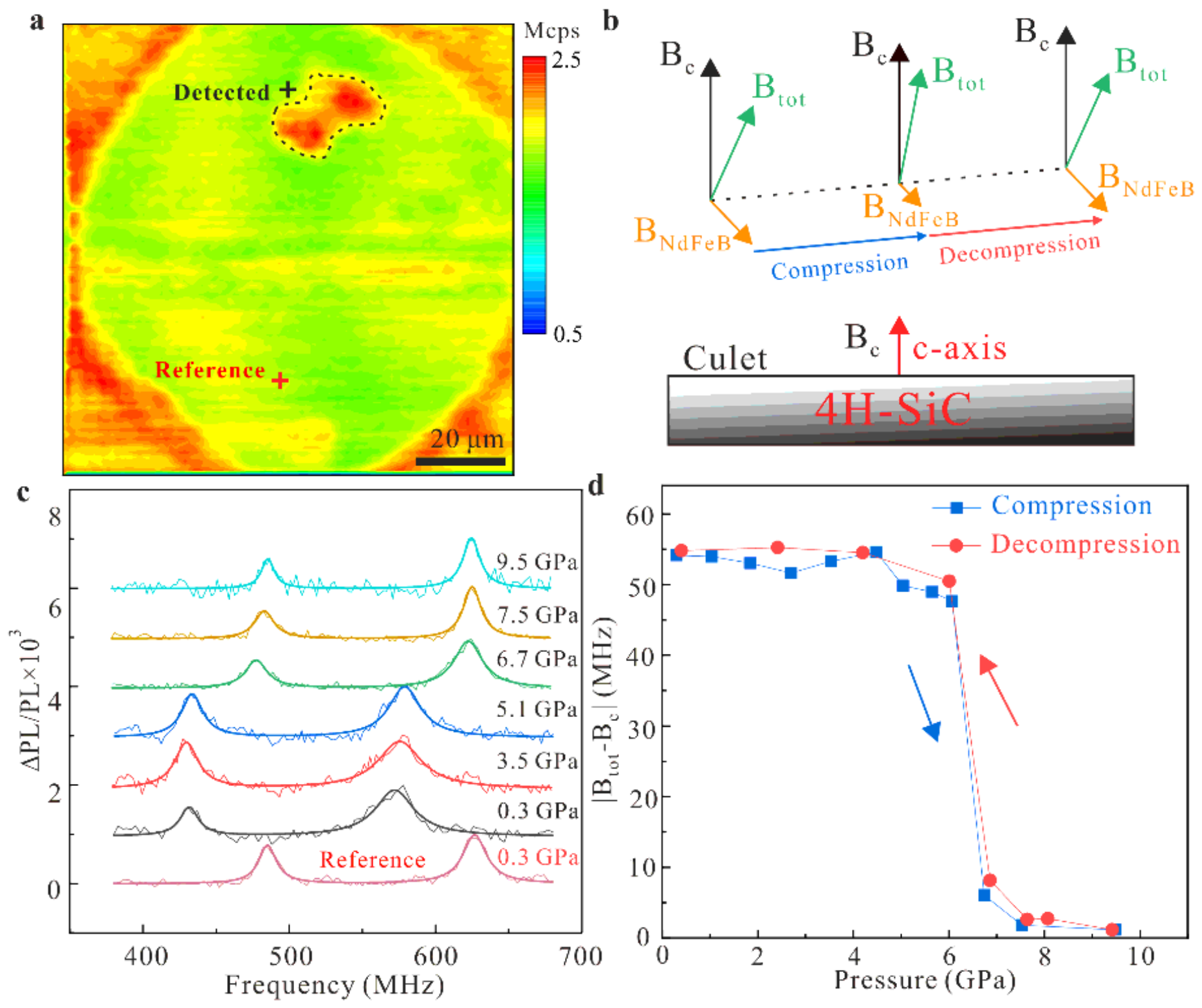

\section{Figure 3}

The detection of pressure-induced magnetic phase transition of a $\mathrm{Nd}_{2} \mathrm{Fe}_{14} \mathrm{~B}$ magnet using the shallow $\mathrm{V}_{\mathrm{Si}}$ defects. a, The PL image of the $\mathrm{V}_{\mathrm{Si}}$ defects and $\mathrm{Nd}_{2} \mathrm{Fe}_{14} \mathrm{~B}$ sample in the culet surface. The black and red crosses are the detected and reference positions, respectively. The black dashed line region indicates the $\mathrm{Nd}_{2} \mathrm{Fe}_{14} \mathrm{~B}$ sample. The scale bar is $20 \mu \mathrm{m}$. b, Three typically local magnetic fields vectors during the pressure-induced magnetic phase transition in the compression and decompression processes. The external magnetic field $B_{C}$ is along the $c$-axis of the $4 \mathrm{H}$-SiC. The magnetic field of the $\mathrm{Nd}_{2} \mathrm{Fe}_{14} \mathrm{~B}$ sample and the total magnetic field are labelled as $B_{N d F e B}$ and $B_{\text {tot }}$, respectively. $c$, The shallow $V_{S i}$ defects ODMR spectra in the detected and reference positions in the culet surface during the compression process. $d$, The inferred magnetic fields of the $\mathrm{Nd}_{2} \mathrm{Fe}_{14} \mathrm{~B}$ sample measured by $\mathrm{V}_{\mathrm{Si}}$ defects during the compression (blue squares) and decompression (red circles) processes, respectively. 

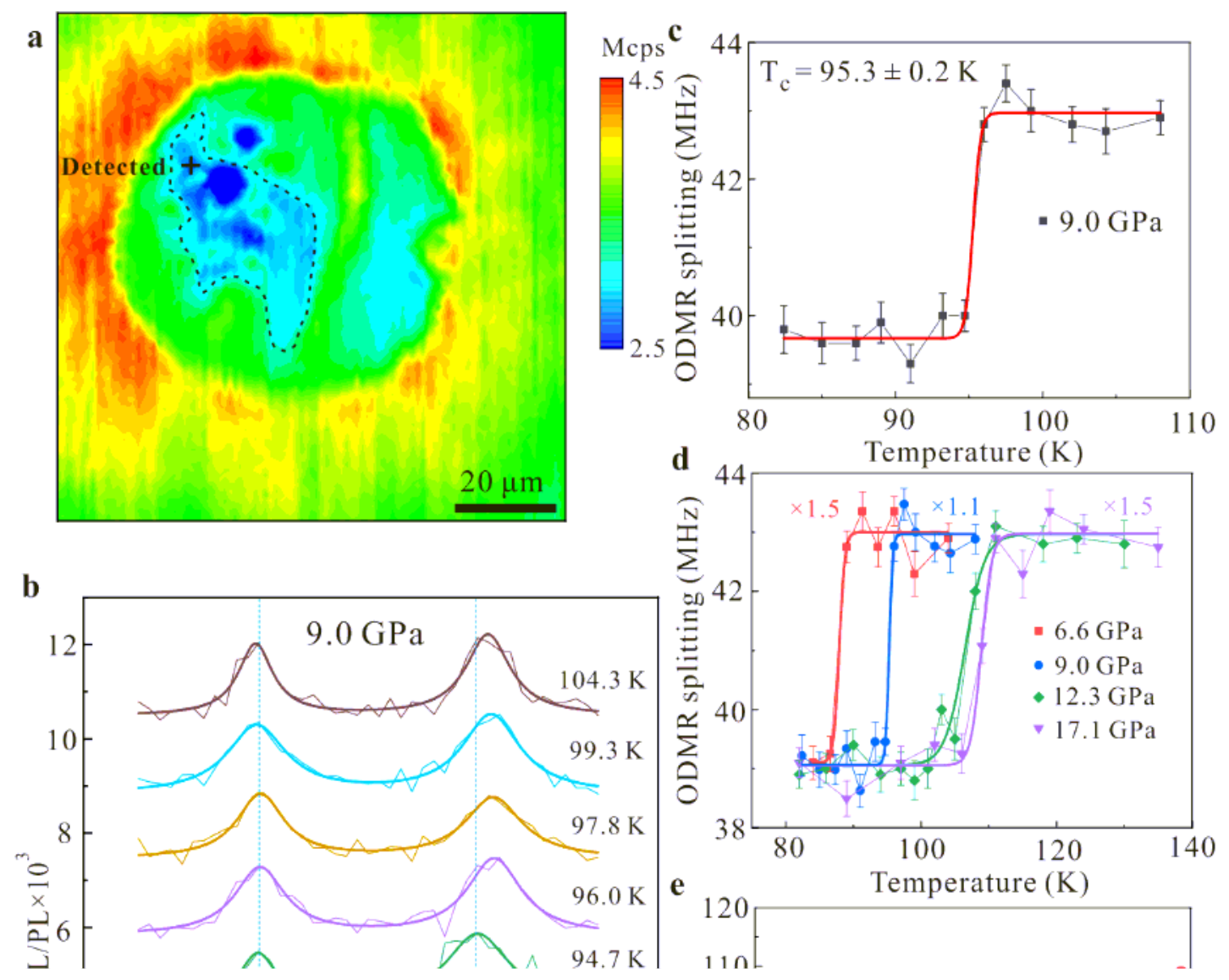

\section{Figure 4}

The measurement of temperature-pressure phase diagram of the superconductor $\mathrm{YBa}_{2} \mathrm{Cu}_{3} \mathrm{O}_{6.66}$ using implanted $\mathrm{V}_{\mathrm{Si}}$ defects. $\mathrm{a}$, The confocal scanning image of $\mathrm{YBa}_{2} \mathrm{Cu}_{3} \mathrm{O}_{6.66}$ sample and $\mathrm{V}_{\mathrm{Si}}$ defects in the culet surface. The black dashed line region marks the investigated $\mathrm{YBa}_{2} \mathrm{Cu}_{3} \mathrm{O}_{6.66}$ sample and the black cross marks the corresponding detected position. The size scale bar is $20 \mu \mathrm{m}$. $\mathbf{b}$, The ODMR spectra with the superconductivity diamagnetism in the detected position at different temperatures at $9.0 \mathrm{GPa}$. The dashed lines are guides to an eye only. c, The inferred ODMR splitting during the sample superconducting phase transition at $9.0 \mathrm{GPa}$. The red line is the fitting of the data. The superconducting transition temperature $T_{c}$ is deduced to be $95.2 \pm 0.2 \mathrm{~K}$. $d$, The inferred ODMR splittings as a function of the temperature under different pressures. The labeled coefficients are the ODMR splitting magnification times in order to normalization the data. $\mathbf{e}$, $\mathrm{The} \mathrm{YBa}_{2} \mathrm{Cu}_{3} \mathrm{O}_{6.66} \mathrm{~T}_{\mathrm{c}}$-pressure phase diagram. The $\mathrm{T}_{\mathrm{C}}$ under ambient pressure (blue dot) is measured through a magnetic property measurement system (see Supplementary Note 2 for more details). The $T_{C}$ under pressures (red dots) are inferred from the ODMR splittings. The shadow area represents the superconducting state and the transparent area is the normal state for the $\mathrm{YBa}_{2} \mathrm{Cu}_{3} \mathrm{O}_{6.66}$. The error bars are obtained from the fitting standard deviations are smaller than the symbol sizes.

\section{Supplementary Files}

This is a list of supplementary files associated with this preprint. Click to download. 
- SupplementaryXXinformationXXforMagneticdetectionunderhighpressureusingdesignedsiliconvacancycentersinsiliconcarbide.docx 